\title{
A MAXIMALLY PATHOLOGICAL BROUWER HOMEOMORPHISM
}

\author{
EDWARD WARWICK DAW
}

\begin{abstract}
This paper constructs a Brouwer homeomorphism which does not act properly discontinuously on any nonempty invariant closed connected set in $\mathbb{R}^{2}$.
\end{abstract}

\section{BACKGROUND}

A Brouwer homeomorphism, $h$, is an orientation preserving homeomorphism of the plane which has no fixed point. Such homeomorphisms can be surprisingly more complex than simple translation, and, as the example presented here demonstrates, quite pathological. Before getting into the gory details of pathological Brouwer homeomorphisms, we will review a few classical results of Brouwer Theory, as presented in [B2] and [G].

Recall that a translation arc for $x \in \mathbb{R}^{2}$ is an arc $\alpha \subset \mathbb{R}^{2}$ joining $x$ to $h(x)$ such that $\alpha \cap h(\alpha)=\{h(x)\}$. Furthermore, for any Brouwer homeomorphism, every point $x \in \mathbb{R}^{2}$ has a translation arc. Brouwer's Lemma says that for any Brouwer homeomorphism, $h$, and any translation arc, $\alpha$, for $h, h^{n}(\alpha) \cap \alpha=\varnothing$ for $|n|>1$. Thus, $A=\bigcup_{n=-\infty}^{\infty} h^{n}(\alpha) \subset \mathbb{R}^{2}$ is an embedded line, called a translation line. We will say that a homeomorphism, $h: \mathbb{R}^{2} \rightarrow \mathbb{R}^{2}$, acts properly discontinuously on a set $B \subset \mathbb{R}^{2}$ if for every compact set $K \subset B$, the set $\left\{n \in \mathbb{Z} \mid h^{n}(K) \cap K \neq \varnothing\right\}$ is finite. Note that $K$ need not be connected. Also, note that translation acts properly discontinuously on all sets.

In simple translation (say $(x, y) \rightarrow(x+1, y)$ ), a translation line, $A$, is a proper subset of $\mathbb{R}^{2}$ with the following four nice properties:

(1) $A$ is closed.

(2) $A$ is connected.

(3) $A$ is invariant.

(4) $A$ is acted upon properly discontinuously.

In fact, any translation line which is properly embedded has these four properties. However, in the general case, $A$ is not necessarily properly embedded, so $A$ may not be closed.

Note that we can always find subsets for a Brouwer homeomorphism with any three of the above four properties. Any translation arc, $\alpha$, contains no limit points of $A-\alpha$, other than the end points of $\alpha$ for sequences in $h(\alpha)$ and $h^{-1}(\alpha)$, and there is an $\epsilon$-neighborhood of $\alpha$ which is "translated" along with

Received by the editors January 27, 1992.

1991 Mathematics Subject Classification. Primary 58F13, 54H20; Secondary 57N05, 54G20. 
$\alpha$ (see e.g. [B1]). Thus $A$ always has properties (2), (3), and (4). Furthermore, the closure of $A$ always has properties (1), (2), and (3). A result related to Brouwer's lemma is: If $h$ is a Brouwer homeomorphism, and $x \in \mathbb{R}^{2}$, then the orbit of $x, O(x)=\left\{h^{n}(x): n \in \mathbb{Z}\right\}$ has no accumulation points in $\mathbb{R}^{2}$. Thus, the orbit of a point has properties (1), (3), and (4). A single point has properties (1), (2), and (4).

Main Result. There is a Brouwer homeomorphism, $h: \mathbb{R}^{2} \rightarrow \mathbb{R}^{2}$, which fails to act properly discontinuously on any proper closed connected invariant subset.

By the facts presented above, no attribute can be eliminated from the preceding sentence. Such a Brouwer homeomorphism is maximally pathological in the sense that it acts unlike translation on all closed connected invariant sets. This answers the question raised by Brown, Slaminka, and Transue in [BST], where they present an example of a Brouwer homeomorphism which has no closed invariant line.

For illustrative purposes, we will first consider a relatively simple Brouwer homeomorphism, then complicate it to get a Brouwer homeomorphism with almost the properties we want, and finally complicate that Brouwer homeomorphism to get one which has all the desired properties. The construction of these examples, particularly the second, was inspired by [BST], although familiarity with that paper is not assumed here.

\section{Simple eXAMPle}

The simple example, $h_{1}$, is a composition of two homeomorphisms, $T$ and $S_{1}$, where $T$ is unit left translation, and $S_{1}$ is described below.

$S_{1}$ preserves the leaves of the foliation of $\mathbb{R}^{2}$ by the lines $y=x+b$ where $b \in \mathbb{R}$. It fixes the integer points along the $x$-axis, and all points sufficiently far away from these fixed points will move 2 units to the right in the $x$-direction (over 2 and up 2 since $S_{1}$ preserves leaves). Between the integer points on the $x$-axis and the points which are sufficiently far away, we just "fill in" continuously (being sure not to introduce any more fixed points). (See Figure 1.)

Note that $h_{1}=T S_{1}$ is essentially translation with some squeezing and stretching. However, $h_{1}$ is interesting in that the behavior of its orbits fall into four distinct classes. First is the unique orbit consisting of the integer points in the $x$-axis, which moves from right to left. Second are the orbits of points between the leaves which contain the integer points. These points primarily move from the lower left to the upper right, although they may take a slight detour right to left for a few iterations near the $x$-axis. Third are orbits of points on the bottom half of leaves which contain integer points. These points start in the lower left, move up and right until they near the integer points on the $\mathrm{x}$-axis where they move out to the left, getting asymptotically close to the integer points on the $x$-axis. Finally, the orbits of points in the top half of the leaves containing the integer points start near the integer points on the $x$-axis to the right, move to the left, and then get away from the integer points on the $\mathrm{x}$-axis, moving up and to the right.

While the opposing left-to-right and right-to-left movement appears interesting, it all "goes off to $\infty$ " (i.e., any compact set containing right moving and left moving points is eventually mapped outside any compact set), and, as we noted above, we are left with what is basically translation. What we need is 


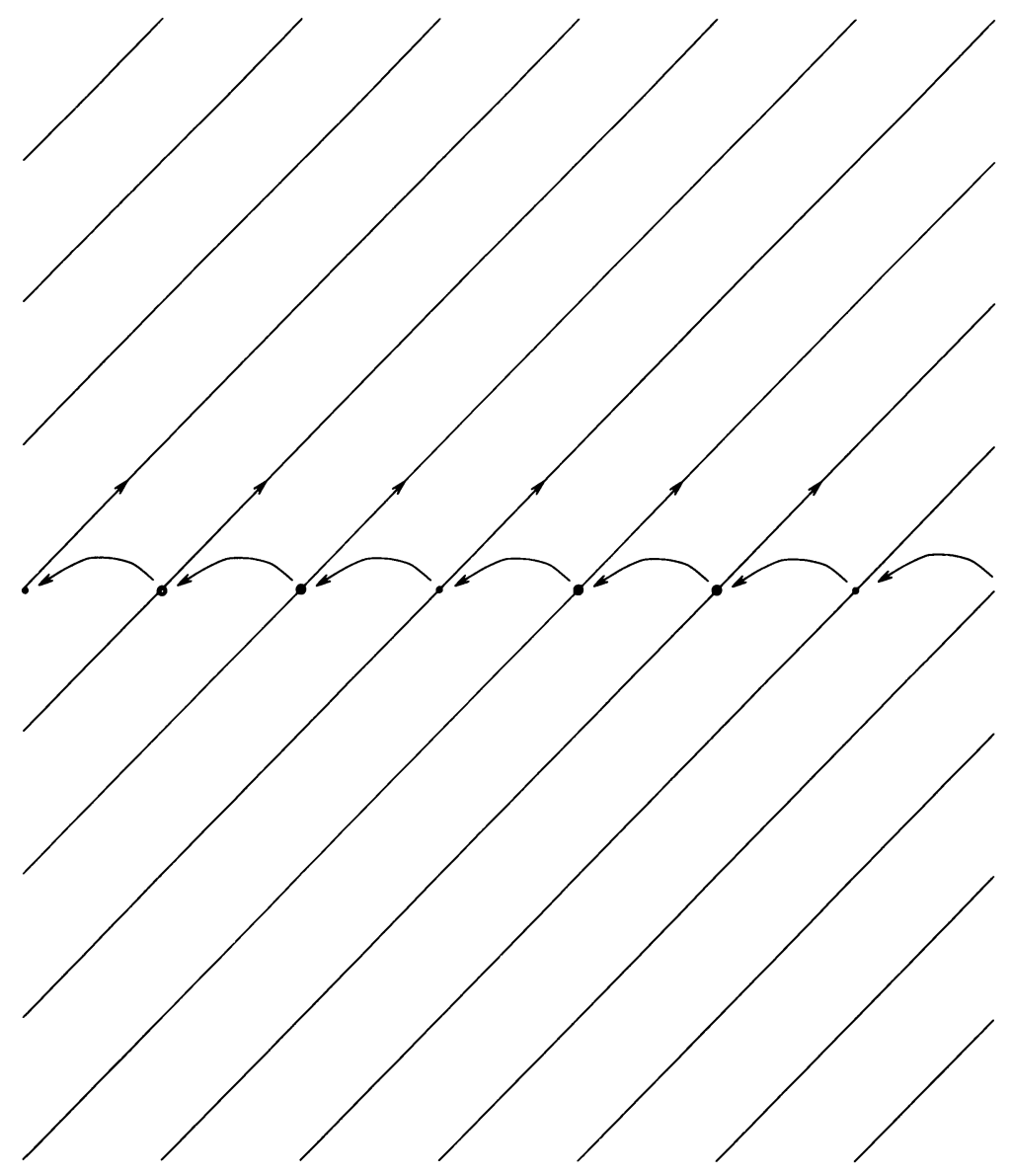

FIGURE 1

some way to "bound" this behavior so it does not "escape." One way to do this is to "shove spikes" from the top and bottom into the foliation we used for $S_{1}$. This produces the second example, $h_{2}$, which fails to admit a nonempty closed compactly connected invariant subset upon which $h_{2}$ acts properly discontinuously.

\section{DEFINITIONS OF SECOND AND THIRD EXAMPLES}

When we say a set, $M$, is compactly connected, we mean that any two points in $M$ are contained in a connected compact subset of $M$.

To define the second example, $h_{2}$, we need to make the concept of "shoving in spikes" precise. The spikes are the set $S=\{(x, y) \mid x \in \mathbb{Z}, y \leq-1$ or $y \geq 1\}$. We define a map $R: \mathbb{R}^{2} \rightarrow\left(\mathbb{R}^{2}-S\right)$ which preserves vertical lines by $R((x, y))=\left(x,|2 \operatorname{frac}(x)-1|(y /|y|)\left(1-2^{-|y|}\right)+(1-|2 \operatorname{frac}(x)-1|) y\right)$ if $y \neq 0$, and $R((x, y))=(x, 0)$ if $y=0 . R$ sends the foliation which we used for $h_{1}$ (Figure 1) to one which looks like Figure 2. Let $g_{\lambda}$ be the leaf of this foliation which intersects the $x$-axis at $-\lambda$.

We can define $h_{2}$ by $h_{2}=R h_{1} R^{-1}$ on $\mathbb{R}-S$ and by $h_{2}((x, y))=(x+1, y)$ 


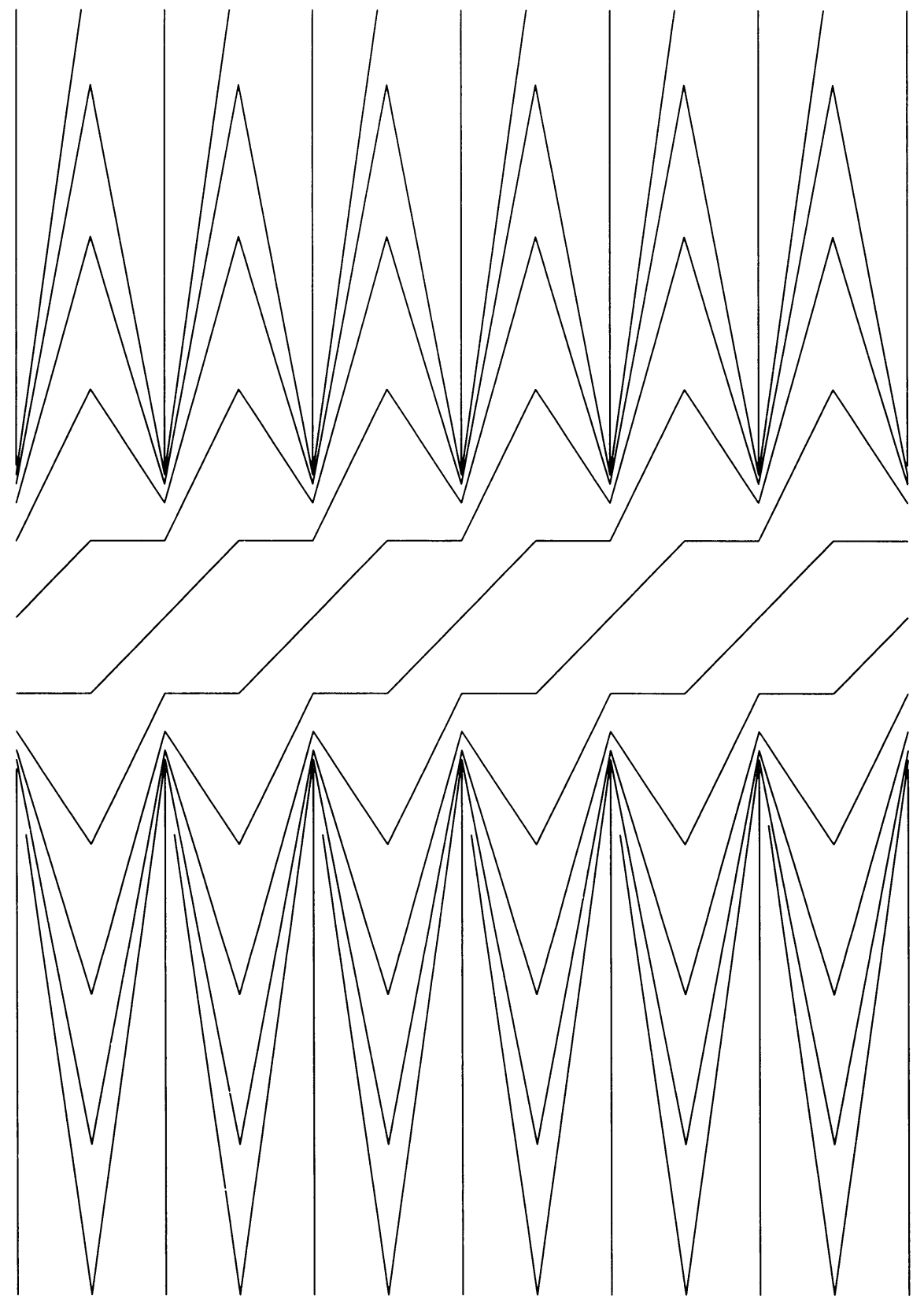

FIGURE 2

on $S$. As we shall see, $h_{2}$ has no proper closed compactly connected invariant subset of $\mathbb{R}^{2}$ which is acted upon properly discontinuously.

To complicate $h_{2}$ into the final example, we compose $h_{2}$ with a homeomorphism, $D$, made up of infinitely many nonoverlapping Dehn twists, i.e., $h_{3}=D h_{2} . h_{3}$ will not act properly discontinuously on any proper closed connected invariant set.

We will construct $D$ as follows: Let $d_{0}$ be a Dehn twist which acts on the topological annulus, $A_{0}$, which surrounds an $\epsilon$-neighborhood $(\epsilon<<1)$ of 


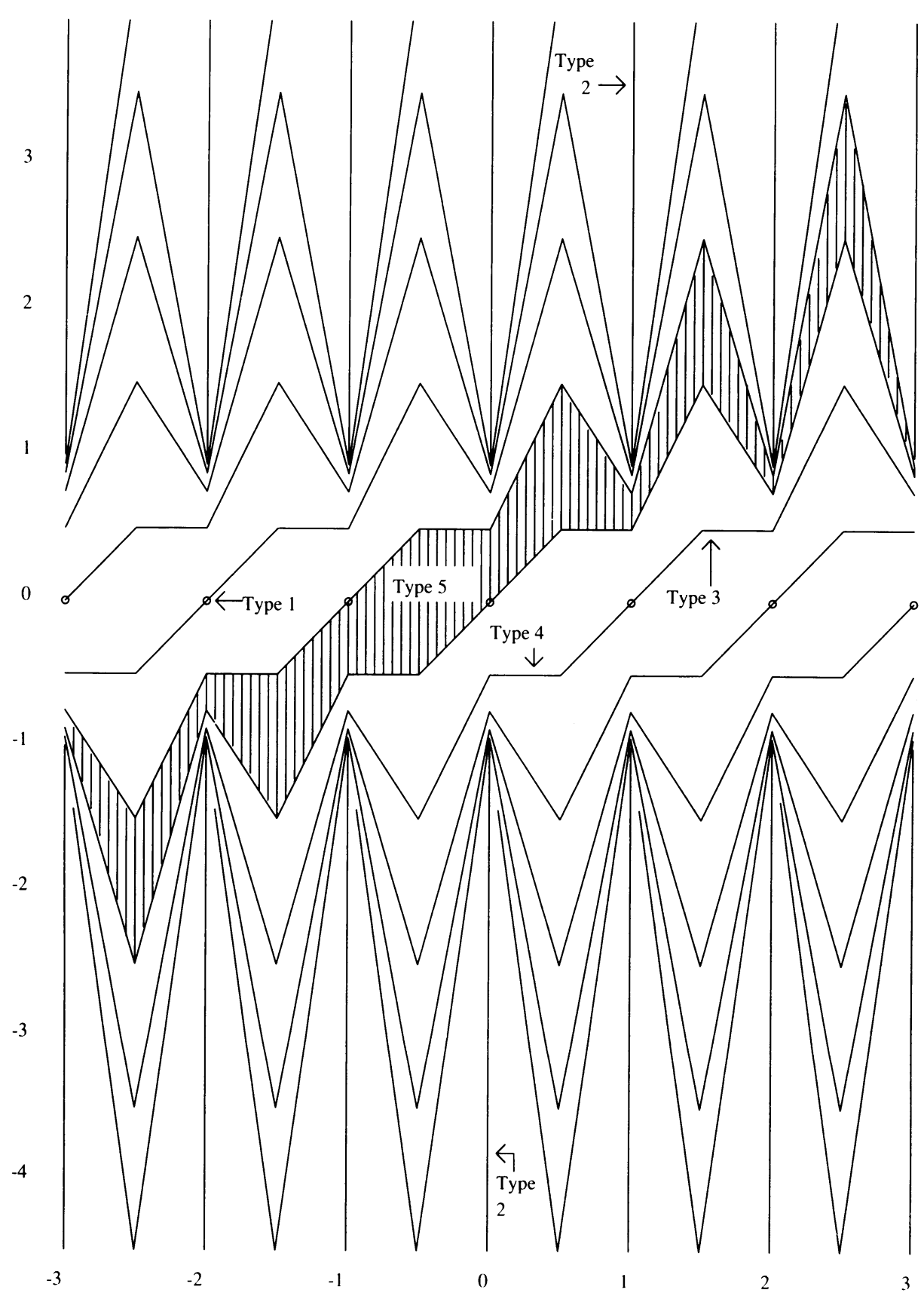

FIGURE 3

the segment $[-2,2]$ on $g_{0} ; A_{0}$ is contained in a $2 \epsilon$-neighborhood of this segment, and has "thickness" $\delta<\epsilon$. Let $A_{i}$ and $d_{i}, i \in \mathbb{Z}$, be the right and left translates of $A_{0}$ and $d_{0}$ such that $A_{i} \cap g_{i} \neq \varnothing . D$ is defined to be the infinite composition of the $d_{i}$ 's, i.e., $\left.D\right|_{A_{i}}=d_{i}$ and $D$ is the identity elsewhere.

To get a better picture of the dynamics of $h_{2}$ (and consequently the dynamics of $h_{3}$ ), look at the following regions, which we call fundamental (after [A]) (see Figure 3): 
(1) Each integer point on the $x$-axis.

(2) Each spike $\{(x, y): x=n, y \geq 1\}$ or $\{(x, y): x=n, y \leq-1\}$ where $n \in \mathbb{Z}$.

(3) The right half of each integer leaf of the foliation, $g_{n}, n \in \mathbb{Z}$, with $x>-n$.

(4) The left half of each integer leaf of the foliation, $g_{n}, n \in \mathbb{Z}$, with $x<-n$.

(5) Each region between two successive integer leaves of the foliation, $g_{n}$ and $g_{n+1}, n \in \mathbb{Z}$.

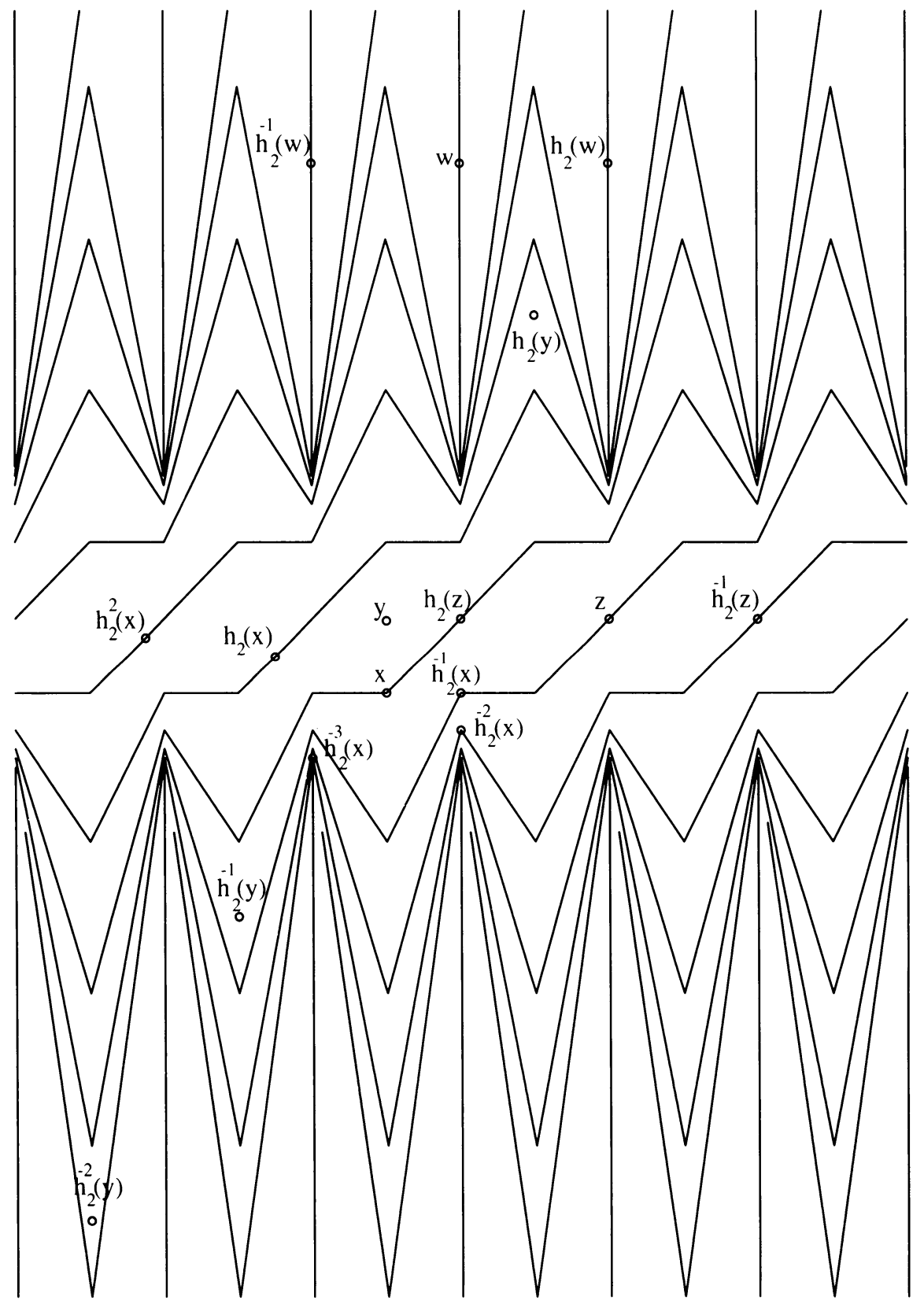

FIGURE 4 
Now, examine the images of points in these regions under successive forward and backward iterations of $h_{2}$ (see Figure 4). Note that no region is setwise fixed, and each is taken to another of the same type. Points that make up a region of type 1 move from right to left under forward iterations, and the union of all such points, which we will call $P$, is setwise fixed and closed. Points in regions of type 2 move from left to right, and the union of the spikes in the top half-plane, which we will call $M$, is setwise fixed and closed, as is the union of the spikes in the bottom half-plane, which we will call $N$. Points in regions of type 3 eventually move to the right under both forward and backward iterations of $h_{2}$. Under forward iterations, these points converge to the set $M$, i.e., given an $x$ in a region of type 3 , for all $\epsilon>0$, there exists $n \in \mathbb{Z}$ such that $\left(h_{2}\right)^{m}(x)$ is within the $\epsilon$-neighborhood of $M \cup\{(x, y) \mid y \geq 1 / \epsilon\}$ for all $m>n$. Under backward iterations, these points converge to the set $P$. Similarly, points in regions of type 4 move to the left under both positive and negative iterations of $h_{2}$. Under positive iterations, these points converge to the set $P$. Under negative iterations, these points converge to the set $N$. Points in regions of type 5 eventually move from left to right under forward iterations of $h_{2}$. Under positive iterations, such a point will converge to the set $M$, and under negative iterations, it will converge to the set $N$.

The infinitely many Dehn twists we composed $h_{2}$ with to get $h_{3}$ cause there to be infinitely many different types of fundamental regions for $h_{3}$. As a result, rather than doing a detailed analysis of each type of fundamental region, we will simply note that, away from the very thin Dehn twists which make up $D$, $h_{3}$ behaves the same as $h_{2}$.

\section{KEY ARGUMENT}

We will say that a set, $I$, has $x$-divergent points under a Brouwer homeomorphism, $h$, if there are points $w, z \in I$ such that either the $x$-coordinate of $h^{n}(w)$ goes to $+\infty$ and the $x$-coordinate of $h^{n}(z)$ goes to $-\infty$ as $n \rightarrow+\infty$ or the $x$-coordinate of $h^{n}(w)$ goes to $+\infty$ and the $x$-coordinate of $h^{n}(z)$ goes to $-\infty$ as $n \rightarrow-\infty$. The key idea in showing that $h_{2}$ and $h_{3}$ have the stated properties is contained in the following two claims:

Claim 1 . If $I$ is a compact connected set in $\mathbb{R}^{2}-S$, and $I$ has $x$-divergent points under $h_{2}$ (or $h_{3}$ ), then $h_{2}^{n}(I)$ (or $h_{3}^{n}(I)$ ) intersects, for infinitely many $n$, any vertical segment connecting a top spike to a bottom spike, i.e., $V_{m}=$ $(x, y): x=m,-1<y<1$, where $m \in \mathbb{Z}$.

Proof. Since $h_{2}$ and $h_{3}$ restricted to $\mathbb{R}^{2}-S$ are homeomorphisms of $\mathbb{R}^{2}-S$ to $\mathbb{R}^{2}-S, h_{2}^{n}(I) \subset \mathbb{R}^{2}-S$ and $h_{3}^{2}(I) \subset \mathbb{R}^{2}-S$. Furthermore, since $h_{2}$ and $h_{3}$ are continuous and $I$ is connected, $h_{2}(I)$ and $h_{3}(I)$ are connected. Each $V_{m}$ separates $\mathbb{R}^{2}-S$. Since $I$ contains $x$-divergent points, $h_{2}^{n}(I)$ (or $h_{3}^{n}(I)$ ) must contain a point on either side of each $V_{m}$ for some $n$, and thus, since the $V_{m}$ separate $\mathbb{R}^{2}-S$, intersect that $V_{m}$. Without loss, assume that $I$ contains $x$-divergent points as $n \rightarrow+\infty$. Then if $h_{2}^{N}(I)$ (or $h_{3}^{N}(I)$ ) has a "left moving" point, $z$, to the left of some $V_{m}$ and a "right moving" point to the right of the same $V_{m}$, then $h_{2}^{n}(I)$ (or $\left.h_{3}^{n}(I)\right)$ must intersect that $V_{m}$ for all $n>N$ (see Figure 5). Thus we have infinitely many images of $h_{2}^{n}(I)\left(\right.$ or $\left.h_{3}^{n}(I)\right)$ intersecting $V_{m}$. 


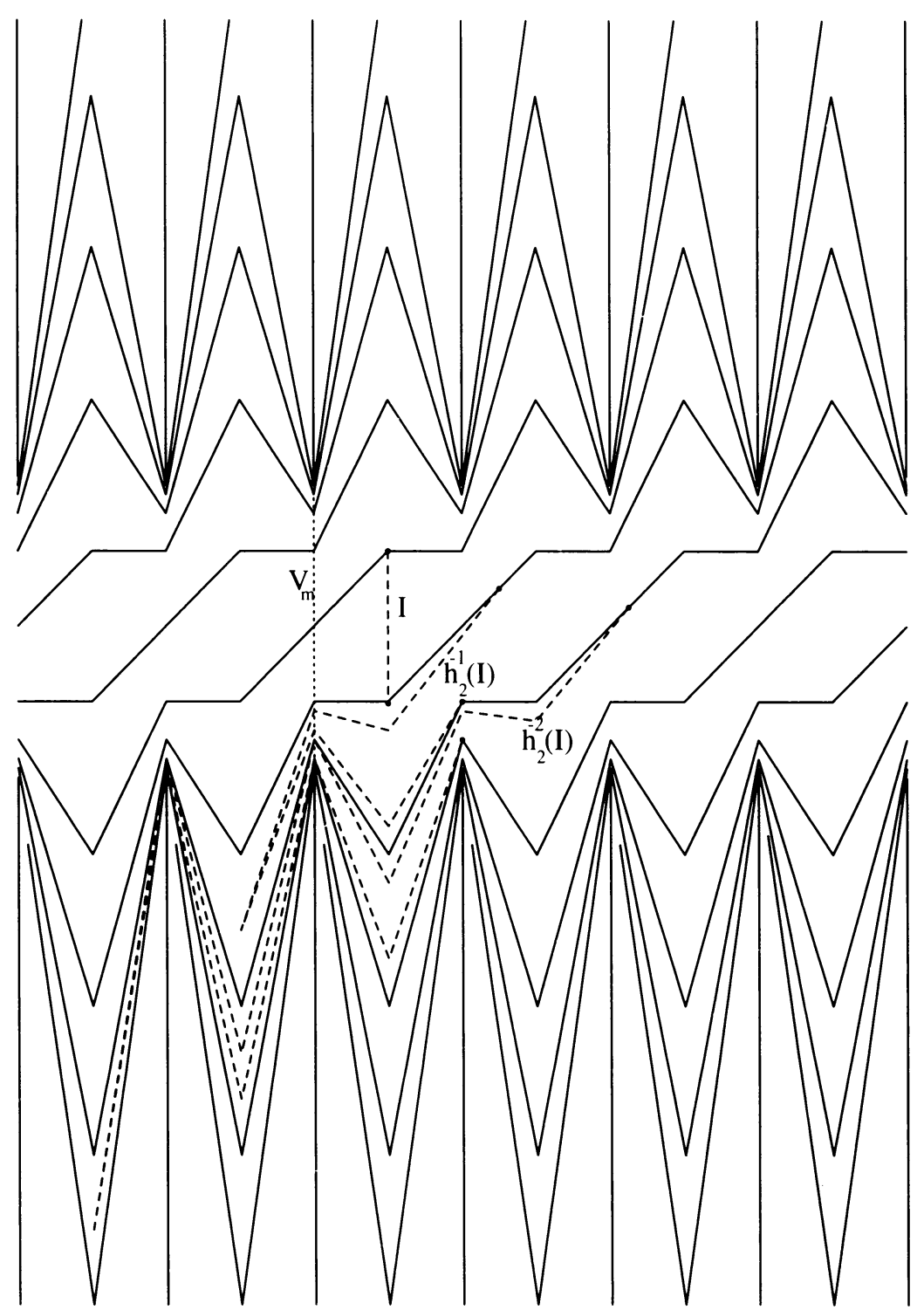

FIGURE 5

Claim 2. If a compact connected $I$ with $x$-divergent points is contained in a closed invariant (under $h_{2}\left(\right.$ or $\left.h_{3}\right)$ ) subset, $J$, then $h_{2}$ (or $h_{3}$ ) cannot act properly discontinuously on $J$

Proof. Since $J$ is invariant, and $I \subset J$, all the images of $I$ are in $J$. If we take a compact set, $K \subset \mathbb{R}^{2}$ such that some $V_{m} \subset K$, then $K \cap J$ is compact (since $J$ is closed). So $I \cup(K \cap J)$ is a compact subset of $J$. Since $h_{2}^{n}(I) \cap K \neq \varnothing$ for infinitely many $n$, and $h_{2}^{n}(I) \cap K \subset J, h_{2}^{n}(I \cup(K \cap J)) \cap(I \cup(K \cap J)) \neq \varnothing$ for infinitely many $n$. Thus $h_{2}$ does not act properly discontinuously on $J$. The argument is the same for $h_{3}$. 


\section{THE COMPACTLY CONNECTED CASE}

It is now left to show that all closed compactly connected invariant subsets under $h_{2}$ and all closed connected invariant subsets under $h_{3}$ contain a compact connected set, $I$, with $x$-divergent points. We will first consider the case where our closed invariant set, $J$, is compactly connected.

Note that while $h_{2}$ preserves the foliation by $g_{\lambda}$ 's shown in Figure 2 and translates the leaves, $h_{3}$ does not preserve this foliation. $h_{3}$ does, however, preserve the leaves outside a $2 \epsilon$-neighborhood of the leaves with integer subscripts (the integer leaves), and translates them. For, example, both $h_{2}$ and $h_{3}$ take $g_{1 / 2}$ to $g_{3 / 2}$. We will call the leaves, $g_{n+1 / 2}$, half way between two successive integer leaves, $g_{n}$ and $g_{n+1}$, half-integer leaves.

Claim 3. Any connected set, $C$, in $\mathbb{R}^{2}$ which contains points on both sides of a properly embedded strip, $B$ (e.g. $\left\{(x, y) \in \mathbb{R}^{2} \mid 0 \leq x \leq 1\right\}$ ), must contain a connected subset $C^{\prime} \subset C \cap B$ which contains a point in $C$ on a side of $B$ and a point in the interior of $B$.

Proof. Consider the connected components of $C \cap B$ and $C \backslash($ interior $B)$. These components form a set of connected subsets $\left\{C_{\alpha}\right\}$ such that $\cup C_{\alpha}=C$. Note that each $C_{\alpha}$ must contain a point in one of the sides of $B$. Suppose that no $C_{\alpha}$ contains a point in the interior of $B$. Then $C$ is disjoint since we assumed it contained points on both sides of $B$. We can let $C^{\prime}$ be any $C_{\alpha}$ which contains a point in the interior of $B$.

Claim 4. Any nonempty connected invariant set, $J$, under $h_{2}$ (or $h_{3}$ ) must contain a point on every half-integer leaf (in fact, every leaf) of the foliation by $g_{\lambda}$ 's.

Proof. If $J$ contains a point on a half-integer leaf, $g_{n+1 / 2}$, then the image under $h_{2}$ of that point is on $g_{n+3 / 2}$ and the preimage is on $g_{n-1 / 2}$, so iteration gives us a point on every half-integer leaf. If $J$ contains a point between two half-integer leaves, the images of that point must also be in $J$. But the images of that point must be between different pairs of half-integer leaves, so since there is some half-integer leaf between any two images of such a point and the leaf divides the plane into two parts, $J$ must have a point on a half-integer leaf. If $J$ contains a point in a region of type 2, again, the images of that point, which are in different regions of type 2 , must also be in $J$. But each region of type 2 is disconnected from all other regions of type 2 , so $J$ must contain a point outside of a region of type 2. Thus $J$ always has a point on each half-integer leaves. Since any other leaf separates half-integer leaves, $J$ must contain a point on every leaf.

Claim 5. If $J$ is a compactly connected closed invariant set under $h_{2}$ (or $h_{3}$ ), then there is a $I \subset J$ such that $I$ is compact, connected, and contains $x$ divergent points.

Proof. By Claim 4, there are points in $J$ on successive half-integer leaves, $g_{n-1 / 2}$ and $g_{n+1 / 2}$. Since $J$ is compactly connected, we can let $I$ be a compact connected set containing points in $g_{n-1 / 2}$ and $g_{n+1 / 2}$. On the leaves $g_{n-1 / 2}$ and $g_{n+1 / 2}, h_{2}$ (and $h_{3}$ ) increases the $x$-coordinate of every point by 1 under each positive iteration (and therefore decreases the $x$-coordinate of every point 
in these sets by 1 under each negative iteration). Thus, for $w \in g_{n-1 / 2}$ or $w \in g_{n+1 / 2}$, the $x$ coordinate of $h_{i}^{m}(w)$ goes to $+\infty$ as $m \rightarrow+\infty$ for $i=2,3$, and the $x$-coordinate of $h_{i}^{m}(w)$ goes to $-\infty$ as $m \rightarrow-\infty$ for $i=2,3$. So, we need to show that there is some other point, $z \in I$ such that the $x$-coordinate of $h_{i}^{m}(z)$ goes to $-\infty$ as $m \rightarrow+\infty$ or the $x$-coordinate of $h_{i}^{m}(z)$ goes to $+\infty$ as $m \rightarrow-\infty$ for $i=2,3$.

First we will consider $h_{2}$. Note that since $g_{n}$ separates $g_{n-1 / 2}$ and $g_{n+1 / 2}$, $I$ must contain a point on $g_{n}$. Under $h_{2}, g_{n}$ is composed of points of type 1 , type 3 , and type 4 . As we noted in $\S 3$, if $z$ is of type 1 or type 4 , the $x$-coordinate of $h_{2}^{m}(z)$ goes to $-\infty$ as $m \rightarrow+\infty$, and if $z$ is of type 1 and type 3 , the $x$-coordinate of $h_{2}^{m}(z)$ goes to $+\infty$ as $m \rightarrow-\infty$. Thus $I$ contains $x$-divergent points under $h_{2}$.

Now, we consider what happens when $J$ is invariant under $h_{3}$. The idea is the same as in the $h_{2}$ case: there is some set between $g_{n-1 / 2}$ and $g_{n+1 / 2}$ which separates them and consists of points which are $x$-divergent from the points in $g_{n-1 / 2}$ and $g_{n+1 / 2}$ under $h_{3}$. The set is, however, more complicated than $g_{n}$.

Let $B_{n}$ be the bounded component of $\mathbb{R}^{2}-A_{n}$. Consider $B_{n} \cap g_{n}$. We can divide this set into three parts, $B g_{n}^{1}$, the point on the $x$-axis (which is of type 1 under $h_{2}$ ), $B g_{n}^{3}$, the points above the $x$-axis (which were of type 3 under $h_{2}$ ), and $B g_{n}^{4}$, the points below the x-axis (which were of type 4 under $\left.h_{2}\right)$. Now, one can easily see that $h_{3}^{i}\left(B g_{n}^{1} \cup B g_{n}^{3}\right) \subset B_{n+i} \cap g_{n+i}$ for $i<0$ and $h_{3}^{i}\left(B g_{n}^{1} \cup B g_{n}^{4}\right) \subset B_{n+i} \cap g_{n+i}$ for $i>0$, thus these points are $x$-divergent from those in $g_{n+1 / 2}$ and $g_{n-1 / 2}$. However, it is not only these points, but the points in $B_{k} \cap g_{k}$ for $k \in \mathbb{Z}$ and all the images of these points, $h_{3}^{j}\left(B_{k} \cap g_{k}\right)$ for $j \in \mathbb{Z}$, which are $x$-divergent from $g_{n+1 / 2}$ and $g_{n-1 / 2}$. In particular, the set $\bigcup_{i=-\infty}^{+\infty} h_{3}^{-} i\left(B_{n+i} \cap g_{n+i}\right)$ separates $g_{n+1 / 2}$ and $g_{n-1 / 2}$ and consists of points which are $x$-divergent from the points in these two leaves. Thus, any compact connected set which contains points in both $g_{n+1 / 2}$ and $g_{n-1 / 2}$ must contain $x$-divergent points under $h_{3}$.

Thus Claim 2 and Claim 5 directly imply the following:

Initial Result. $h_{2}$ and $h_{3}$ do not act properly discontinuously on any nonempty closed compactly connected invariant subset of $\mathbb{R}^{2}$.

Note that invariance under $h_{2}$ and $h_{3}$ are not equivalent.

\section{CONNECTED BUt NOT COMPACTLY CONNECTED SETS}

Now, let us consider what connected but not compactly connected sets look like. The example to keep in mind is the fish bone set (see Figure 6), $F=$ $A \cup B \cup C \cup D \cup E$, where

(1) $A=\{$ segments from $(0, n)$ to $(1-1 / n, 0)$ for $n \in \mathbb{Z}, n \geq 2\}$,

(2) $B=\{$ segments from $(0, n)$ to $(1 / n-1,0)$ for $n \in \mathbb{Z}, n \geq 2\}$,

(3) $C=\{(0, y) \mid y \in \mathbb{R}, y \geq 0\}$,

(4) $D=\{(1, y) \mid y \in \mathbb{R}, y \geq 0\}$,

(5) $E=\{(-1, y) \mid y \in \mathbb{R}, y \geq 0\}$.

We have the following claim about the structure of closed connected but not compactly connected sets: 


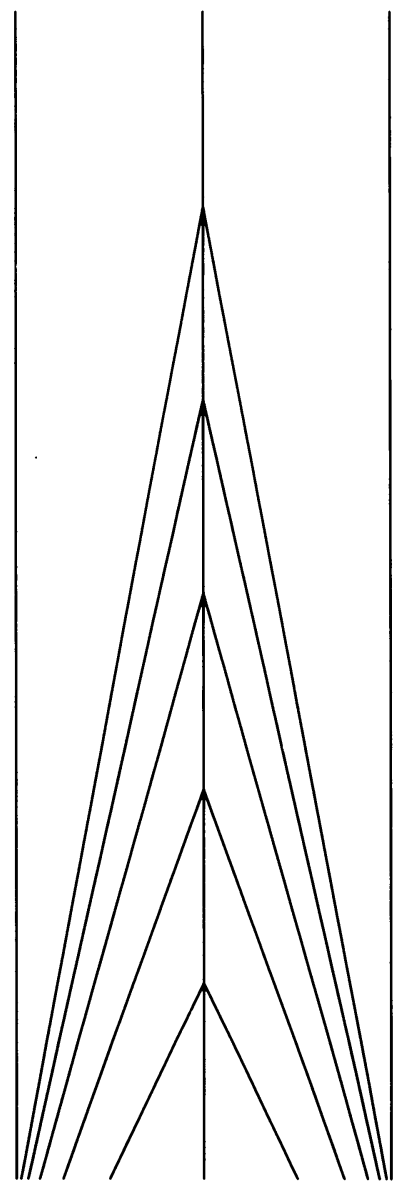

FIGURE 6

Claim 6. Closed connected but not compactly connected sets are composed of disjoint unbounded maximal compactly connected subsets, called ray sets.

Proof. Clearly, if $J$ is a connected but not compactly connected set, we may separate $J$ into compactly connected components (points are compactly connected). Any two maximal compactly connected components must be disjoint because if $X$ and $Y$ are two such sets and $z \in X \cap Y \neq \varnothing$ then for any $x \in X$ and $y \in Y$, there are compact subsets $X^{\prime} \subset X$ and $Y^{\prime} \subset Y$ which connect $x$ to $z$ and $y$ to $z$. So $X^{\prime} \cup Y^{\prime}$ is a compact set which connects $x$ to $y$.

Let $J_{0}$ be a maximal compactly connected component of a closed connected but not compactly connected set, $J$, and suppose that $J_{0}$ is bounded. If $\left\{x_{i}\right\}$ is a sequence in $J_{0}$ with accumulation point $x_{0}$, then, since $J$ is closed, $x_{0} \in$ $J$, and, since $J_{0}$ is bounded and compactly connected and $J_{0} \cup x_{0}$ must be connected, $J_{0}$ must contain $x_{0}$. Thus $J_{0}$ is closed.

Now, since $J$ is connected and $J \neq J_{0}$, there must be a sequence $\left\{y_{i}\right\}$ not in $J_{0}$ which converges to a point in $J_{0}$. We will divide the ways this sequence can converge into three cases.

Case 1. Suppose $\left\{y_{i}\right\}$ is contained in some other maximal compactly connected component, $J_{1}$ (see Figure 7A). Let $K$ be the set of points, $x$, such that there are sequences in both $J_{0}$ and $J_{1}$ which converge to $x$. If any connected 
component, $K^{\prime}$, of $K$ is compact, then $J_{0} \cup K^{\prime}$ and $J_{1} \cup K^{\prime}$ are compactly connected and so $J_{0} \cup J_{1} \cup K^{\prime}\left(=J_{0} \cup J_{1}\right)$ is compactly connected. But this contradicts $J_{0}$ maximal. If no component of $K$ is bounded, we get a contradiction of $J_{0}$ bounded, since $J_{0}$ is closed.

Case 2. We may assume that each point of the sequence $\left\{y_{i}\right\}$ is on a different compactly connected component of $J$. Suppose that infinitely many $y_{i}$ 's are in unbounded compactly connected components. We may then assume that each $y_{i}$ is in an unbounded $J_{i}$ (see Figure 7B). Let us look at an $\epsilon$-neighborhood and a $2 \epsilon$-neighborhood of $J_{0}$. Let $L_{i}$ be the connected component of $J_{i} \cap 2 \epsilon$ neighborhood of $J_{0}$ which contains $y_{i}$. Now the $L_{i}$ are compact connected sets which are all contained in a compact set, so they must converge to a compact connected set, $L_{0}$. Since we know the $L_{i}$ contain a sequence which converges to a point in $J_{0}, L_{0}$ must be in $J_{0}$. However, by a modification of Claim 3 for the annulus, all $L_{i}$ must contain points in the $2 \epsilon$-neighborhood minus the $\epsilon$-neighborhood, and therefore so must the convergence set, giving us a contradiction.

Case 3. We may assume that for every sequence, $\left\{y_{i}\right\}_{\alpha}$, each point of that sequence is on a different bounded compactly connected component. Consider all sequences $\left\{y_{i}\right\}_{\alpha}$ with each point on a different component, $J_{i_{\alpha}}$, which converge to a point in $J_{0}$. We will say $J_{i_{\alpha}}$ is closer to $J_{0}$ than $J_{k_{\beta}}$ if there is an $\epsilon$-neighborhood of $J_{0}, N_{\epsilon}\left(J_{0}\right)$, for some $\epsilon$ such that $J_{i_{\alpha}} \cap N_{\epsilon}\left(J_{0}\right) \neq \varnothing$ and $J_{k_{\beta}} \cap N_{\epsilon}\left(J_{0}\right)=\varnothing$. If neither $J_{i_{\alpha}}$ nor $J_{k_{\beta}}$ is closer to $J_{0}$ than the other, we will say $J_{i_{\alpha}}$ is as close as $J_{k_{\beta}}$ to $J_{0}$. We need the following subclaim to complete this case.

Subclaim. For every $\epsilon>0$, there is a $J_{I_{\alpha}}$ such that $J_{I_{\alpha}} \subset N_{\epsilon}\left(J_{0}\right)$ and if $J_{i_{\beta}}$ is either closer to $J_{0}$ than $J_{I_{\alpha}}$ or as close as $J_{I_{\alpha}}$ to $J_{0}$, then $J_{i_{\beta}} \subset N_{\epsilon}\left(J_{0}\right)$.

Proof of subclaim. Suppose there is no such $J_{I_{\alpha}}$. Then there is a sequence of $J_{i_{\beta}}$ 's which contains a sequence of points with an accumulation point in $J_{0}$ and a sequence with an accumulation point outside of $N_{\epsilon}\left(J_{0}\right)$. By passing to convergent subsequences and applying reasoning similar to that in case 2 , this gives a contradiction.

Pick an $\epsilon$ and let $J_{I_{\alpha}}$ be a component as in the above claim (see Figure 7C). Let $L=J_{0} \cup J_{I_{\alpha}} \cup\left\{\right.$ all $J_{i_{\beta}}$ closer to $J_{0}$ than $\left.J_{I_{\alpha}}\right\}$. Note that $L \subset N_{\epsilon}\left(J_{0}\right)$ and that there is a $\delta<\epsilon$ such that $J \cap N_{\delta}\left(J_{0}\right) \subset L$.

$L$ is closed because each $J_{i_{\alpha}}$ is closed and all the $J_{i_{\beta}}$ components are compact and connected and all contained in an $\epsilon$-neighborhood of $J_{0}$, so any sequence of $J_{i_{\beta}}$ 's which converges must converge to a compact connected set, $K$, in $N_{\epsilon}\left(J_{0}\right)$. Now, since $J$ is closed, $K \subset J$. Furthermore, since every $J_{i_{\beta}}$ contains a point closer than or as close as $J_{I_{\alpha}}$ to $J_{0}$, there must be a sequence with an accumulation point as close as or closer than $J_{I_{\alpha}}$. But this accumulation point must be in $K$, so $K \subset L$. Thus $L$ is compact.

Now in a compact set, the compactly connected components are the connected components, so $J_{0}, J_{I_{\alpha}}$, and the $J_{i_{\beta}}$ 's are connected components of $L$. Connected components have arbitrarily small closed and open neighborhoods around them, so, in particular, there must be such a neighborhood around $J_{0}$ in $L$. But $N_{\delta}\left(J_{0}\right) \cap J \subset L$, so there is also such a neighborhood around $J_{0}$ in $J$. But then $J_{0}$ is disconnected from the rest of $J$, contradicting the connectedness of $J$. 


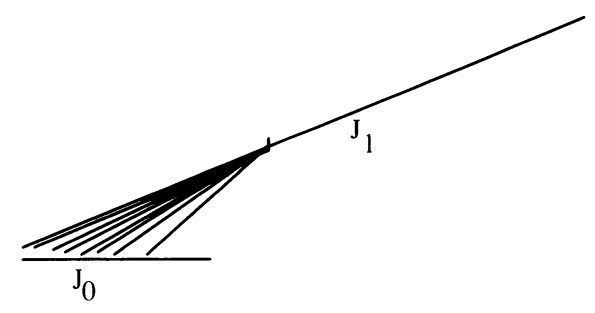

(A)

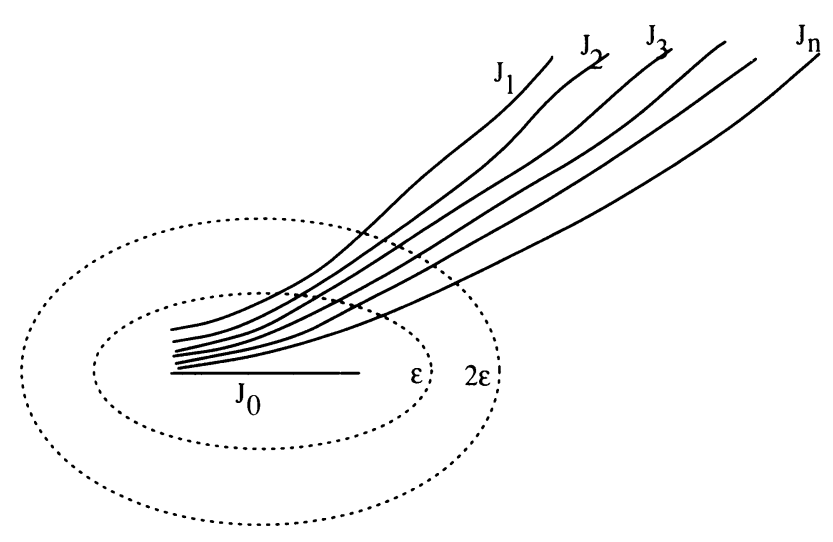

(B)

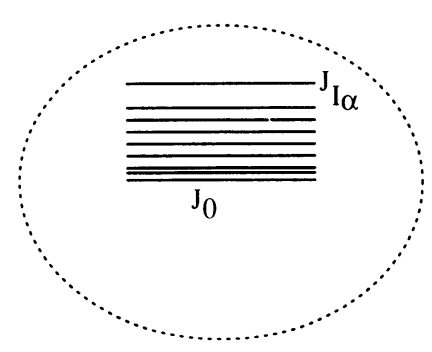

(C)

FIGURE 7

Thus there can be no bounded compactly connected components in a closed connected but not compactly connected set.

\section{Proof of MAIN Result}

Now that the structure of connected not compactly connected sets has been established, we are ready to prove the main result:

Main Result. $h_{3}$ does not act properly discontinuously on any nonempty closed invariant subset, $J$, of $\mathbb{R}^{2}$.

Proof. As before, Let $B_{i}$ be the bounded component of $\mathbb{R}^{2}-A_{i}$, and let $X_{i}$ be the integer point on the $x$-axis contained in $B_{i}$. Initially, suppose $J$ contains a point, $z$, inside some $B_{j}$ on the integer leaf, $g_{j}$. Now, either $J$ is compactly connected or $z$ is in some ray set $J_{i} \subset J$ which is compactly connected and 

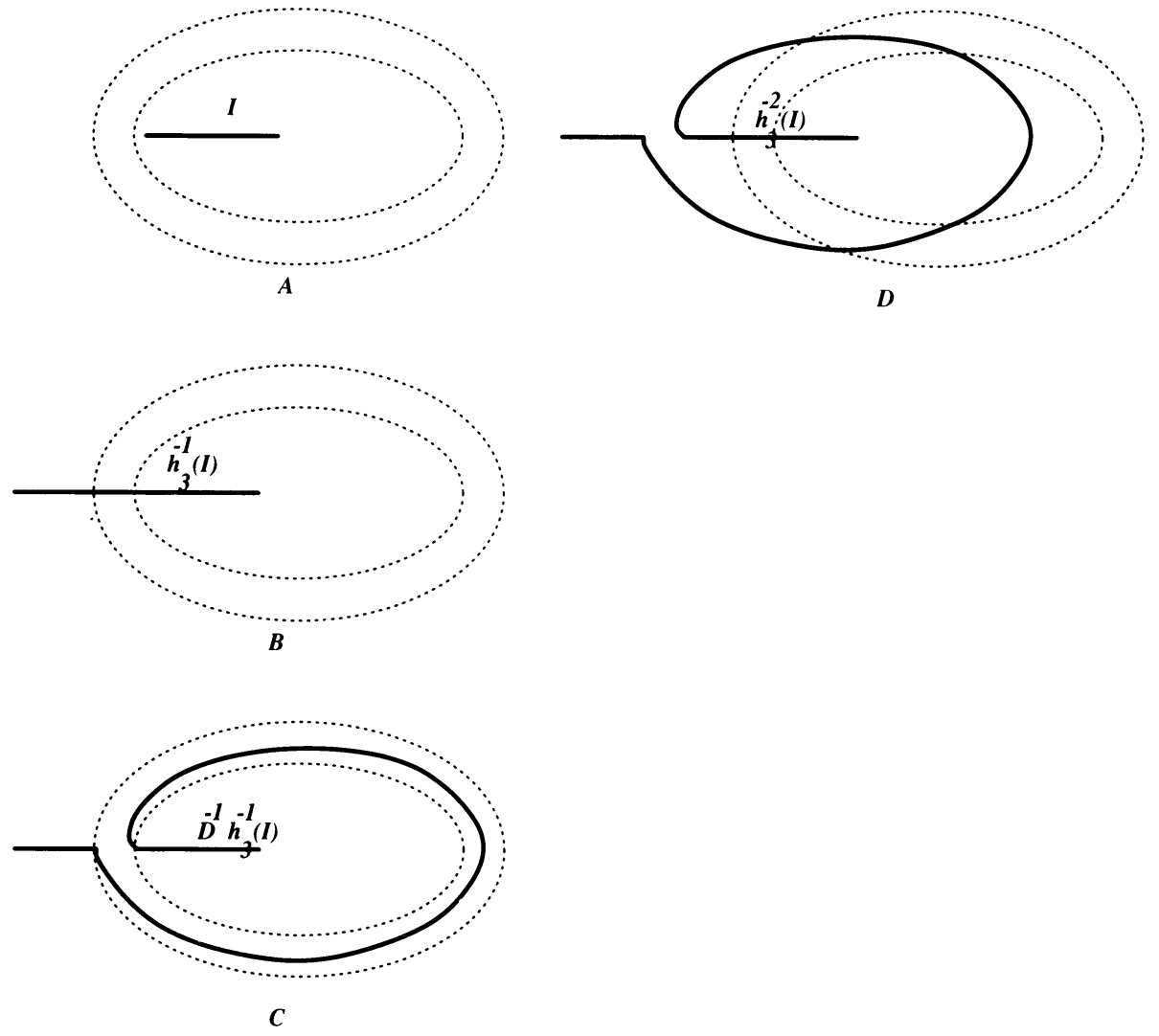

Figure 8

unbounded. Either way, $z$ must be compactly connected to other points in $B_{j}$, i.e., there is a compact connected subset, $I$, of $J$ in $B_{j}$ which contains $z$ and other points in $B_{j}$. The proof breaks into two cases, depending on whether or not $I \subset g_{j}$.

Case 1. Suppose there is a compactly connected $I \subset J \cap B_{j}$ containing $z$ and a point not in $g_{j}$. Without loss, assume that $z$ is on the bottom half of $g_{j}$ and consider forward iterations of $h_{3}$. By Claim 3, we may assume that $I$ is within a $1 / 2 \epsilon$-neighborhood of $g_{j} . h_{3}^{n}(z)$ goes to the left (has decreasing $x$-coordinate) as $n \rightarrow+\infty$, while the points, $w$, not on $g_{j}$ will slowly move across the $B_{k}$ 's with $w-X_{i}$ increasing until $h_{3}^{n}(I)$ intersects the boundary of some $B_{j+n}$ within $1 / 2 \epsilon$ of $g_{j+n}$ on the right side of $B_{j+n}$. Now this section of the boundary of $B_{j+n}$ is mapped out of $B_{j+n+1} \cup A_{j+n+1}$ and to the right under $h_{3}$, and all further images of $I$ will contain $w$ 's further to the right, with $x$-coordinates $\rightarrow+\infty$ as $n \rightarrow+\infty$. So $I$ contains $x$-divergent points, and eventually gets stretched to the left and right under $h_{3}$.

Case 2. Suppose we have a $z \in B_{j} \cap g_{j}$, but there is no compactly connected set in $B_{j}$ for any $j$ connecting a $z$ to a point in $B_{j}-g_{j}$. Then, since $J$ is compactly connected or $z \in J_{i}$, where $J_{i}$ is some ray set, $J$ must contain a "segment" $I$ on $g_{j}$ which connects $z$ to the boundary of $B_{j}, \partial B_{j}$ (otherwise 
we would have a compactly connected component). Since we are assuming that there is no $I$ as in case 1 , we may choose $I$ and $z$ so that $h_{3}^{n}(z) \in B_{j+n}$ for $-1 \leq n \leq 1$. Without loss, say that $I$ connects $z$ to $\partial B_{j} \cap g_{j}$ on the bottom half of $g_{j}$ (see Figure 8A) and look at negative iterations of $h_{3}$. Note that $h_{3}^{-1}=h_{2}^{-1} D^{-1} \cdot h_{3}^{-1}(I)$ is contained in $g_{j-1}$ and crosses $A_{j-1}$ (see Figure 8B). $D^{-1} h_{3}^{-1}(I) \subset g_{j-1} \cup A_{j-1}$ and it runs from $z$ to $\partial B_{j-1}$ along $g_{j-1}$, wraps once around $A_{j-1}$, intersecting $g_{j-1}$ on its top half, and leaves $A_{j-1}$ along the bottom half of $g_{j-1}$ (see Figure 8C). Now, the point at which $D^{-1} h_{3}^{-1}(I)$ intersects the top half of $g_{j-1}$ is mapped to a point in $B_{j-2} \cap g_{j-2}$ on the top half of $g_{j-2}$ by $h_{2}^{-1}$ and the points in $D^{-1} h_{3}^{-1}(I)$ near this intersection not on $G_{j-1}$ are mapped to points nearby inside $B_{j-2}$. Thus $h_{3}^{-2}(I)$ contains a compact connected set containing a point in $B_{j-2} \cap g_{j-2}$ and a point in $B_{j-2}-g_{j-2}$ (see Figure 8D), which contradicts our assumptions and reduces us to the previous case.

Thus, if $h_{3}$ is to act properly discontinuously on $J$, we must have $J \cap\left(B_{j} \cap\right.$ $\left.g_{j}\right)=\varnothing$ for all $j$, so suppose now that this is the case. Now, since $J$ is invariant, we must have $J \cap\left(\bigcup_{i=-\infty}^{+\infty} \bigcup_{j=-\infty}^{+\infty} h_{3}^{i}\left(B_{j} \cap g_{j}\right)=\varnothing\right.$. But $\bigcup_{i=-\infty}^{+\infty} h_{3}^{-i}\left(B_{i} \cap g_{i}\right)$ divides the plane into two unbounded regions, and, more importantly, separates leaves, $g_{\lambda}$ (for $|\lambda-\operatorname{int}(\lambda)|>2 \epsilon$ ), from their images. Thus, if $\mathrm{J}$ is a connected closed invariant set, it must contain a point in this set. Thus $h_{3}$ does not act properly discontinuously on any nonempty closed connected invariant subset.

\section{REFERENCES}

[A] S. A. Andrea, On homeomorphisms of the plane which have no fixed points, Abh. Math. Sem. Univ. Hamburg 30 (1967), 61-74.

[B1] M. Brown, Homeomorphisms of two-dimensional manifolds, Houston J. Math. 11 (1985), 455-469.

[B2] A A new proof of Brouwer's lemma on translation arcs, Houston I. Math. 10 (1984), $35-41$.

[BST] M. Brown, E. E. Slaminka, and W. Transue, An orientation preserving fixed point free homeomorphism of the plane which admits no closed invariant line, Topology Appl. 29 (1988), 213-217.

[G] Lucien Guillou, Le theoreme de translation plane de Brouwer: une demonstration simplifiee menant a une nouvelle preuve du theoreme de Poincare-Birkhoff; preprint.

Department of Mathematics, University of California, Los Angeles, 405 Hilgard Avenue, Los ANGeles, CA 90024

Current address: Department of Psychiatry, Washington University, 4940 Children's Place, St. Louis, Missouri 63110

E-mail address: warwick@bacchus . wust 1 . edu 